\section{Addison, Sir Thomas}

M. Bidlingmaier

Medizinische Klinik und Poliklinik IV, Klinikum der

Universität München, München, Deutschland

Lebensdaten Geboren im April 1793 in Long Benton, Newcastle-upon-Tyne; gestorben am 29. Juni 1860 in Brighton, East Sussex. Englischer Arzt. Medizinstudium 1812-1815, seit 1817 am Guy's Hospital in London. 1838 Wahl zum Fellow des Royal College of Physicians, seit 1840 Professor. Ärztliche und wissenschaftliche Tätigkeit bis 1860. Addison litt unter Depressionen. Tod durch Suizid.

Verdienste $\mathrm{Zu}$ Lebzeiten geschätzter Arzt und einflussreicher akademischer Lehrer. Aus heutiger Sicht war sein wich- tigster Beitrag die Erstbeschreibung der später nach ihm benannten primären Nebennierenrindeninsuffizienz (Morbus Addison). Addison arbeitete insgesamt 11 Fälle dieser damals in ca. $90 \%$ der Fälle durch eine Tuberkulose verursachten Erkrankung auf. Weniger bekannt ist, dass Addison bereits 1843 die heute als perniziöse Anämie bekannte Erkrankung als besondere Form der Anämie beschrieben hatte.

\section{Literatur}

Addison T (1849) Chronics suprarenal insufficiency, usually due to tuberculosis of suprarenal capsule. Lond Med Gazette 43:517-518

Pearce JMS (2004) Thomas Addison (1793-1860). J R Soc Med 97(6):297-300 